\title{
TOPOLOGICAL PROPERTIES OF LOCALIZATIONS, FLAT OVERRINGS AND SUBLOCALIZATIONS
}

\author{
DARIO SPIRITO
}

\begin{abstract}
We study the set of localizations of an integral domain from a topological point of view, showing that it is always a spectral space and characterizing when it is a proconstructible subspace of the space of all overrings. We then study the same problems in the case of quotient rings, flat overrings and sublocalizations.
\end{abstract}

\section{INTRODUCTION}

The Zariski topology on the set Over $(D)$ of overrings of an integral domain was introduced as a natural generalization of the Zariski topology on the space $\operatorname{Zar}(D)$ of valuation overrings of $D$ (called the Zariski space of $D$ ), which in turn was introduced by Zariski in order to tackle the problem of resolution of singularities [35, 36.

It has been proved that $\operatorname{Over}(D)$, like $\operatorname{Zar}(D)$, is a spectral space, meaning that it is homeomorphic to the prime spectrum of a ring [10, Proposition 3.5]. There are other subspaces of Over $(D)$ that are always spectral: for example, this happens for the space of integrally closed overrings [10, Proposition 3.6] and the space of local overrings [12, Corollary 2.14].

In the last two cases, the role of $D$ in the definition of the space is merely to provide a setting $(\operatorname{Over}(D))$ : that is, for an overring, being integrally closed or local (or a valuation domain, for the case of $\operatorname{Zar}(D)$ ) is a property completely independent from $D$. Indeed, with very similar proofs it is possible to generalize these results to the case of the spaces of rings comprised between two fixed rings (see e.g. [10, Propositions 3.5 and 3.6] and [12, Example 2.13]), as well as using these methods to study spaces of modules [31, Example 2.2].

In this paper, we study four subspaces of $\operatorname{Over}(D)$ that are much more closely related to $D$; more precisely, such that, given an overring $T$, the belonging of $T$ to the space depends not on the properties of $T$ but rather on the relation between $D$ and $T$. In Section 3 we shall

Date: July 9, 2021.

2010 Mathematics Subject Classification. 13A15, 13B30, 13B40, 13C11, $13 \mathrm{G} 05$.

Key words and phrases. Localizations; flat overrings; spectral spaces; constructible topology.

This work was partially supported by GNSAGA of Istituto Nazionale di Alta Matematica. 
start from the space of localizations (at prime ideals); then we will consider the space of quotient rings (Section 4), sublocalizations of $D$ (i.e., intersection of localizations of $D$; Section 5D) and flat overrings (Section 6).

In each case, we will study two questions: under which conditions they are spectral spaces and under which condition they are closed in the constructible topology of Over $(D)$. We shall answer completely these questions in the case of localizations (Theorem 3.2) and quotient rings (Corollary 4.3 and Theorem 4.4); for sublocalizations we will find a sufficient condition (Theorem 5.5 ), while for flat overrings we will prove a characterization that is, however, very difficult to use (Proposition 6.1). We shall also study the space of flat submodules of an $R$-module (for rings $R$ that are not necessarily integral domains) and the possibility of representing the space of sublocalizations of $D$ in a more topological way.

\section{Preliminaries}

2.1. Spectral spaces. A spectral space is a topological space homeomorphic to the prime spectrum of a (commutative, unitary) ring (endowed with the Zariski topology). Spectral spaces can be characterized topologically as those spaces that are $T_{0}$ (i.e., such that for every pair of points at least one of them is contained in an open set not containing the other), compact, with a basis of open and compact subsets closed by finite intersections, and such that every nonempty irreducible closed subset has a generic point (i.e., it is the closure of a single point) [25, Proposition 4].

If $X$ is a spectral space, the constructible topology (or patch topology) on $X$ (which we denote by $X^{\text {cons }}$ ) is the coarsest topology such that the open and compact subspaces of the original topology are both open and closed. The space $X^{\text {cons }}$ is always a spectral space, that is moreover Hausdorff and totally disconnected [25, Theorem 1].

A subset $Y \subseteq X$ is said to be proconstructible if it is closed, with respect to the constructible topology; in this case, the constructible topology on $Y$ coincides with the topology induced by the constructible topology on $X$, and $Y$ (with the original topology) is a spectral space (this follows from [6, 1.9.5(vi-vii)]). The converse does not hold, i.e., a subspace $Y$ of a spectral space $X$ may be spectral but not proconstructible; however, the following result holds.

Lemma 2.1. Let $Y \subseteq X$ be spectral spaces. Suppose that there is a subbasis $\mathcal{B}$ of $X$ such that, for every $B \in \mathcal{B}$, both $B$ and $B \cap Y$ are compact. Then, $Y$ is a proconstructible subset of $X$.

Proof. The hypothesis on $\mathcal{B}$ implies that the inclusion map $Y \hookrightarrow X$ is a spectral map; by [6, 1.9.5(vii)], it follows that $Y$ is a proconstructible subset of $X$. 
For further results about the constructible topology and the relation between ultrafilters and the constructible topology, see [19, 11, 10, 12].

2.2. The space $\mathcal{X}(X)$. Let $X$ be a spectral space. The inverse topology on $X$ is the space $X^{\text {inv }}$ having, as a basis of closed sets, the open and compact subspaces of $X$; equivalently, it is the topology having as closed sets the subsets of $X$ that are compact and closed by generizations. The space $X^{\text {inv }}$ is again a spectral space. Following [15], we denote by $\mathcal{X}(X)$ the space of nonempty subsets of $X$ that are closed in the inverse topology; this space can be endowed with a topology having, as a basis of open sets, the sets of the form

$$
\mathcal{U}(\Omega):=\{Y \in \mathcal{X}(X) \mid Y \subseteq \Omega\}
$$

as $\Omega$ ranges among the open and compact subspaces of $X$. Under this topology, $\mathcal{X}(X)$ is again a spectral space [15, Theorem 3.2(1)].

If $X=\operatorname{Spec}(R)$ for some ring $R$, we set $\mathcal{X}(R):=\mathcal{X}(\operatorname{Spec}(R))$.

2.3. Semistar operations. Let $D$ be an integral domain with quotient field $K$, and let $\mathbf{F}(D)$ be the set of $D$-submodules of $K$. A semistar operation on $D$ is a map $\star: \mathbf{F}(D) \longrightarrow \mathbf{F}(D)$ such that, for every $I, J \in \mathbf{F}(D)$ and every $x \in K$,

(1) $I \subseteq I^{\star}$;

(2) $\left(I^{\star}\right)^{\star}=I^{\star}$;

(3) if $I \subseteq J$ then $I^{\star} \subseteq J^{\star}$;

(4) $x \cdot I^{\star}=(x I)^{\star}$.

A semistar operation is called spectral if it is in the form $s_{\Delta}$ for some $\Delta \subseteq \operatorname{Spec}(D)$, where

$$
I^{s_{\Delta}}:=\bigcap\left\{I D_{P} \mid P \in \Delta\right\}
$$

for every $I \in \mathbf{F}(D)$. If $\star$ is spectral, then $(I \cap J)^{\star}=I^{\star} \cap J^{\star}$ for every $I, J \in \mathbf{F}(D)$.

Starting from any semistar operations $\star$, we can define two maps $\star_{f}$ and $\widetilde{\star}$ by putting, for every $I \in \mathbf{F}(D)$,

$$
I^{\star}=\bigcup\left\{J^{\star} \mid J \subseteq I, J \text { is finitely generated }\right\}
$$

and

$$
I^{\widetilde{\star}}:=\bigcup\left\{(I: E) \mid 1 \in E^{\star}, E \text { is finitely generated }\right\} .
$$

Both $\star_{f}$ and $\widetilde{\star}$ are semistar operations, and we always have $\left(\star_{f}\right)_{f}=\star_{f}$ and $\widetilde{\star}=\widetilde{\star}$. If $\star=\star_{f}$ then $\star$ is said to be of finite type; on the other hand, $\star=\widetilde{\star}$ if and only if $\star$ is spectral and of finite type.

If $\star=s_{\Delta}$ is a spectral operation, then $\star$ is of finite type if and only if $\Delta$ is compact [16, Corollary 4.4].

The space $\operatorname{SStar}(D)$ of semistar operations on $D$ can be endowed with a topology having, as a basis of open sets, the sets of the form

$$
V_{I}:=\left\{\star \in \operatorname{SStar}(D) \mid 1 \in I^{\star}\right\},
$$


as $I$ ranges in $\mathbf{F}(D)$. In the induced topology, both the space $\operatorname{SStar}_{f}(D)$ of finite-type operations and the space $\operatorname{SStar}_{f, s p}(D)$ of finite-type spectral operations are spectral (see [16, Theorem 2.13] for the former and [13, Theorem 4.6] for the latter). Moreover, $\operatorname{SStar}_{f, s p}(D)$ is homeomorphic to $\mathcal{X}(D)$ [15, Proposition 5.2].

2.4. The $t$-operation. Let $D$ be an integral domain with quotient field $K$, and let $\star$ be a semistar operation on $D$. If $D^{\star}=D$, then the restriction of $\star$ to the set $\mathcal{F}(D)$ of fractional ideals of $D$ is said to be a star operation on $D$. A classical example of a star operation is the divisorial closure (or $v$-operation), which is defined by $I^{v}:=(D:(D$ : $I)$ ), where $(I: J):=\{x \in K \mid x J \subseteq I\}$; the divisorial closure is the biggest star operation on $D$, in the sense that $I^{\star} \subseteq I^{v}$ for every star operation $\star$ and every $I \in \mathcal{F}(D)$.

The $t$-operation is the finite-type operation associated to the $v$ operation; that is, $t:=v_{f}$. The $t$-operation is the biggest finite-type star operation. The $w$-operation, defined by $w:=\widetilde{t}=\widetilde{v}$, is the biggest spectral star operation of finite type.

If $\star$ is a star operation on $D$, a prime ideal $P$ of $D$ such that $P=P^{\star}$ is said to be a $\star$-prime; the set of all $\star$-primes is called the $\star$-spectrum and is denoted by $\mathrm{QSpec}^{\star}(D)$. If $\star=s_{\Delta}$ is a spectral star operation, then $\operatorname{QSpec}^{\star}(D)=\Delta^{\downarrow}=\{Q \in \operatorname{Spec}(D) \mid Q \subseteq P$ for some $P \in \Delta\}$.

We always have $D=\bigcap\left\{D_{P} \mid P \in \operatorname{QSpec}^{t}(D)\right\}$.

See [20, Chapter 32] for more properties of star operations.

2.5. Overrings. Let $D$ be an integral domain with quotient field $K$. An overring of $D$ is a ring comprised between $D$ and $K$. The space Over $(D)$ of the overrings of $D$ can be endowed with a topology having, as a basis of open sets, the sets of the form

$\mathcal{B}\left(x_{1}, \ldots, x_{n}\right):=\left\{T \in \operatorname{Over}(D) \mid x_{1}, \ldots, x_{n} \in T\right\}=\operatorname{Over}\left(D\left[x_{1}, \ldots, x_{n}\right]\right)$, as $x_{1}, \ldots, x_{n}$ range in $K$. Under this topology, $\operatorname{Over}(D)$ is a spectral space [10, Proposition 3.5].

\section{LocAlizations}

The first space we analyze is the space of localizations of an integral domain $D$ at its primes ideals, which we denote by $\operatorname{Loc}(D)$; that is,

$$
\operatorname{Loc}(D):=\left\{D_{P} \mid P \in \operatorname{Spec}(D)\right\} .
$$

Definition 3.1. Let $D$ be an integral domain. We say that $D$ is radcolon coherent if, for every $x \in K \backslash D$, there is a finitely generated ideal I such that $\operatorname{rad}(I)=\operatorname{rad}\left(\left(D:_{D} x\right)\right)$, i.e., if and only if $\mathcal{D}\left(\left(D:_{D} x\right)\right)$ is compact in $\operatorname{Spec}(D)$ for every $x \in K$.

Obvious examples of rad-colon coherent domains are Noetherian domains or, more generally, domains with Noetherian spectrum. Another 
large class of such domains is the class of coherent domains, i.e., domains where the intersection of two finitely generated ideals is still finitely generated; this follows from the fact that $\left(D:_{D} x\right)=D \cap x^{-1} D$. In particular, this class contains all Prüfer domains [20, Proposition 25.4(1)], or more generally the polynomial rings in finitely many variables over Prüfer domains [22, Corollary 7.3.4]. See the following Example 3.3 for a domain that is not rad-colon coherent.

Theorem 3.2. Let $D$ be an integral domain.

(a) $\operatorname{Loc}(D)$ is a spectral space.

(b) $\operatorname{Loc}(D)$ is proconstructible in $\operatorname{Over}(D)$ if and only if $D$ is radcolon coherent.

Proof. (a) By [7, Lemma 2.4], the map

$$
\begin{aligned}
\lambda: \operatorname{Spec}(D) & \longrightarrow \operatorname{Over}(D) \\
P & \longmapsto D_{P} .
\end{aligned}
$$

is a topological embedding whose image is exactly $\operatorname{Loc}(D)$. In particular, since $\operatorname{Spec}(D)$ is a spectral space, so is $\operatorname{Loc}(D)$.

(b) We first note that

$$
\begin{aligned}
\mathcal{B}(x) \cap \operatorname{Loc}(D) & =\left\{D_{P} \in \operatorname{Loc}(D) \mid x \in D_{P}\right\} \\
& =\left\{D_{P} \in \operatorname{Loc}(D) \mid 1 \in\left(D_{P}: x\right) \cap D\right\}= \\
& =\left\{D_{P} \in \operatorname{Loc}(D) \mid 1 \in\left(D::_{D}: x\right) D_{P}\right\}= \\
& =\left\{D_{P} \in \operatorname{Loc}(D) \mid\left(D:_{D}: x\right) \subsetneq P\right\}=\lambda\left(\mathcal{D}\left(\left(D:_{D} x\right)\right)\right) .
\end{aligned}
$$

Suppose $\operatorname{Loc}(D)$ is proconstructible in $\operatorname{Over}(D)$. Since, for any $x \in$ $K, \mathcal{B}(x)$ is also a proconstructible subspace of $\operatorname{Over}(D)$, then $\mathcal{B}(x) \cap$ $\operatorname{Loc}(D)$ is closed in Over $(D)^{\text {cons; }}$ since the Zariski topology is weaker than the constructible topology, $\mathcal{B}(x) \cap \operatorname{Loc}(D)$ must be compact in the Zariski topology. By the previous calculation, $\mathcal{B}(x) \cap \operatorname{Loc}(D)=$ $\lambda\left(\mathcal{D}\left(D:_{D} x\right)\right)$, and thus $\mathcal{D}\left(\left(D:_{D} x\right)\right)$ must be compact. Hence, $D$ is rad-colon coherent.

Conversely, suppose $D$ is rad-colon coherent. Then, each $\mathcal{B}(x) \cap$ $\operatorname{Loc}(D)$ is compact, and thus $\{\mathcal{B}(x) \cap \operatorname{Loc}(D) \mid x \in K\}$ is a subbasis of compact subsets for $\operatorname{Loc}(D)$; applying Lemma 2.1 we see that $\operatorname{Loc}(D)$ is a proconstructible subset of $\operatorname{Over}(D)$.

As a first use of this theorem, we give an example of a domain that is not rad-colon coherent.

Example 3.3. Let $D$ be an essential domain that is not a $\mathrm{P} v \mathrm{MD}$; that is, suppose that $D$ is the intersection of a family of valuation rings, each of which is a localization of $D$, but suppose that there is a $t$-prime ideal $P$ such that $D_{P}$ is not a valuation ring. Such a ring does indeed exists - see [23].

Let $\mathcal{E}$ be the set of prime ideals $P$ of $D$ such that $D_{P}$ is a valuation domain. Since $D$ is not a $\mathrm{P} v \mathrm{MD}$, not all $t$-primes are in $\mathcal{E}$. Since $\mathcal{E} \subseteq$ 
$\operatorname{QSpec}^{t}(D)$ [27, Lemma 3.17], we thus have $\mathcal{E} \subsetneq \operatorname{QSpec}^{t}(D)$. If $\mathcal{E}$ is compact, then $s_{\mathcal{E}}$ is a semistar operation of finite type on $D$; however, since $D$ is essential (and thus, by definition, $\bigcap\left\{D_{P} \mid P \in \mathcal{E}\right\}=D$ ) we have $D^{s_{\mathcal{E}}}=D$, and thus the restriction of $s_{\mathcal{E}}$ to the fractional ideals of $D$ is a spectral star operation of finite type, which implies that $I^{s \mathcal{E}} \subseteq I^{w}$ for every finite-type operation. In particular,

$$
\mathcal{E}=\operatorname{QSpec}^{s \mathcal{E}}(D) \supseteq \operatorname{QSpec}^{w}(D) \supseteq \operatorname{QSpec}^{t}(D),
$$

and thus $\mathcal{E}=\mathrm{QSpec}^{t}(D)$, a contradiction. Therefore, $\mathcal{E}$ is not compact.

However, $\lambda(\mathcal{E})=\operatorname{Loc}(D) \cap \operatorname{Zar}(D)$; if $\operatorname{Loc}(D)$ were to be proconstructible in $\operatorname{Over}(D)$, so would be $\lambda(\mathcal{E})$ (since $\operatorname{Zar}(D)$ is always proconstructible). But this would imply that $\lambda(\mathcal{E})$ is, in particular, compact, a contradiction. Hence $\operatorname{Loc}(D)$ is not proconstructible in $\operatorname{Over}(D)$, and $D$ is not rad-colon coherent.

There are at least three natural ways to extend $\operatorname{Loc}(D)$ to non-local overrings of $D$.

The first is by considering general localizations of $D$ (which we will call, for clarity, quotient rings), that is, overrings in the form $S^{-1} D$ for some multiplicatively closed subsets $S$ of $D$. We denote this set by $\operatorname{Over}_{\mathrm{qr}}(D)$.

The second is through the set of flat overrings of $D$ (that is, overrings that are flat when considered as $D$-modules). We denote this set by $\operatorname{Over}_{\text {flat }}(D)$.

The third is by considering sublocalizations of $D$, i.e., overrings that are intersection of localizations (or, equivalently, quotient rings) of $D$. We denote this set by $\operatorname{Over}_{\text {sloc }}(D)$.

It is well-known that $\operatorname{Over}_{\mathrm{qr}}(D) \subseteq \operatorname{Over}_{\text {flat }}(D) \subseteq \operatorname{Over}_{\text {sloc }}(D)$, and that both inclusions may be strict. For example, any overring of a Prüfer domain is flat, but it need not be a quotient ring: in the case of Dedekind domains, this happens if and only if the class group of $D$ is torsion [21, Corollary 2.6] (more generally, a Prüfer domain $D$ such that $\operatorname{Over}_{\mathrm{qr}}(D)=\operatorname{Over}_{\text {flat }}(D)$ is said to be a $Q R$-domain - see [20, Section 27] or [18, Section 3.2]). As for sublocalizations that are not flat, we shall give an example later (Example 6.3); see also [24].

In all three cases, a natural question is to ask if (or when) the spaces are spectral, and if (or when) they are proconstructible in $\operatorname{Over}(D)$; moreover, we could ask if there is some construction through which we can represent them. We shall treat the case of quotient rings in Section 4, the case of sublocalizations in Section 5 and the case of flat overrings in Section 6.

A first result is a relation between their proconstructibility and the proconstructibility of $\operatorname{Loc}(D)$.

Proposition 3.4. Let $D$ be an integral domain. If $\operatorname{Over}_{\mathrm{qr}}(D)$ or $\operatorname{Over}_{\text {flat }}(D)$ is proconstructible, then $D$ is rad-colon coherent. 
Proof. Let $X$ be either $\operatorname{Over}_{\mathrm{qr}}(D)$ or $\operatorname{Over}_{\text {flat }}(D)$, and let LocOver $(D)$ be the space of local overrings of $D$. Then, $X \cap \operatorname{LocOver}(D)=\operatorname{Loc}(D)$; since LocOver $(D)$ is always proconstructible [12, Corollary 2.14], if $X$ is proconstructible so is $\operatorname{Loc}(D)$. By Theorem 3.2(b), it follows that $D$ is rad-colon coherent.

Note that $\operatorname{Over}_{\text {sloc }}(D) \cap \operatorname{LocOver}(D)$ may not be equal to $\operatorname{Loc}(D)-$ see Example 6.3.

\section{QUOTIEnT RINGS}

As localizations at prime ideals of $D$ can be represented through $\operatorname{Spec}(D)$, we can represent quotient rings by multiplicatively closed subsets; more precisely, there is a one-to-one correspondence between $\operatorname{Over}_{\mathrm{qr}}(D)$ and the set of multiplicatively closed subsets that are saturated. For technical reasons, it is more convenient to work with the complements of multiplicatively closed subsets.

Definition 4.1. Let $R$ be a ring (not necessarily a domain). A semigroup prime on $R$ is a nonempty subset $\mathscr{Q} \subseteq R$ such that:

(1) for each $r \in R$ and for each $\pi \in \mathscr{Q}, r \pi \in \mathscr{Q}$;

(2) for all $\sigma, \tau \in R \backslash \mathscr{Q}, \sigma \tau \in R \backslash \mathscr{Q}$;

(3) $\mathscr{Q} \neq R$.

By [30, (2.3)], a nonempty $\mathscr{Q} \subseteq R$ is a semigroup prime of $R$ if and only if it is a union of prime ideals, if and only if $R \backslash \mathscr{Q}$ is a saturated multiplicatively closed subset.

Let $\mathcal{S}(R)$ denote the set of semigroup primes of a ring $R$. As in [30] and in [14], we endow $\mathcal{S}(R)$ with the topology (which we call the Zariski topology) whose subbasic closed sets have the form

$$
\mathcal{V}_{\mathcal{S}}\left(x_{1}, \ldots, x_{n}\right):=\left\{\mathscr{Q} \in \mathcal{S}(R) \mid x_{1}, \ldots, x_{n} \in \mathscr{Q}\right\},
$$

as $x_{1}, \ldots, x_{n}$ ranges in $R$; equivalently, we can consider the subbasis of open sets

$\mathcal{D}_{\mathcal{S}}\left(x_{1}, \ldots, x_{n}\right):=\mathcal{S}(R) \backslash \mathcal{V}_{\mathcal{S}}\left(x_{1}, \ldots, x_{n}\right)=\left\{\mathscr{Q} \in \mathcal{S}(R) \mid x_{i} \notin \mathscr{Q}\right.$ for some $\left.i\right\}$

We collect the properties of this topology of our interest in the next proposition.

Proposition 4.2. [14, Propositions 2.3 and 3.1] Let $R$ be a ring and endow $\mathcal{S}(R)$ with the Zariski topology.

(a) The family $\left\{\mathcal{D}_{\mathcal{S}}(x) \mid x \in R\right\}$ is a basis of compact and open subsets of $\mathcal{S}(R)$, which is closed by intersections.

(b) The set-theoretic inclusion $\operatorname{Spec}(R) \hookrightarrow \mathcal{S}(R)$ is a topological embedding.

(c) $\mathcal{S}(R)$ is a spectral space. 
(d) Suppose $D$ is an integral domain. The map

$$
\begin{aligned}
\lambda_{q r}: \mathcal{S}(D) & \longrightarrow \operatorname{Over}(D) \\
\mathscr{Q} & \longmapsto(R \backslash \mathscr{Q})^{-1} D .
\end{aligned}
$$

is a topological embedding whose image is $\operatorname{Over}_{\mathrm{qr}}(D)$.

In particular, by points (c) and (d) of the previous proposition we get immediately the following result.

Corollary 4.3. Over $_{\mathrm{qr}}(D)$ is a spectral space for every integral domain D.

On the other hand, proconstructibility holds less frequently for $\mathrm{Over}_{\mathrm{qr}}(D)$ than it does for $\operatorname{Loc}(D)$.

Theorem 4.4. Let $D$ be an integral domain with quotient field $K$. Then, $\operatorname{Over}_{\mathrm{qr}}(D)$ is proconstructible in $\operatorname{Over}(D)$ if and only if, for every $x \in K$, the ideal $\operatorname{rad}\left(\left(D:_{D} x\right)\right)$ is the radical of a principal ideal.

Proof. As in the proof of Theorem [3.2, we see that an overring $T$ is in $\mathcal{B}(x) \cap \operatorname{Over}_{\mathrm{qr}}(D)$ if and only if $T=\lambda_{q r}(\mathscr{Q})$ for some semigroup prime $\mathscr{Q}$ not containing $\left(D:_{D} x\right)$. Moreover, we note that a semigroup prime contains an ideal $I$ if and only if it contains the radical of $I$.

Therefore, if each $\operatorname{rad}\left(\left(D:_{D} x\right)\right)$ is the radical of a principal ideal, then each $\mathcal{B}(x) \cap \operatorname{Over}_{\mathrm{qr}}(D)$ is equal to $\lambda_{q r}\left(\mathcal{D}_{\mathcal{S}}(y)\right)$ for some $y \in D$. However, by Proposition 4.2](a), $\mathcal{D}_{\mathcal{S}}(y)$ is compact, and thus so is $\mathcal{B}(x) \cap$ $\operatorname{Over}_{\mathrm{qr}}(D)$; by Lemma 2.1, $\operatorname{Over}_{\mathrm{qr}}(D)$ is proconstructible in $\operatorname{Over}(D)$.

Conversely, suppose there is a $x \in K$ be such that $I:=\operatorname{rad}\left(\left(D:_{D} x\right)\right)$ is not the radical of a principal ideal.

Claim 1: let $y \in D$. Then, $D\left[y^{-1}\right] \in \mathcal{B}(x)$ if and only if $y \in I$.

If $x \in D\left[y^{-1}\right]$, then

$$
1 \in\left(D\left[y^{-1}\right]:_{D\left[y^{-1}\right]} x\right)=\left(D:_{D} x\right) D\left[y^{-1}\right],
$$

since $D\left[y^{-1}\right]$ is flat over $D$.

If now $P \in \mathcal{V}(I)$ (i.e., $I \subseteq P)$, then in particular $\left(D:_{D} x\right) \subseteq P$, and so $P D\left[y^{-1}\right]=D\left[y^{-1}\right]$; it follows that $y \in P$. Since this happens for every $P \in \mathcal{V}(I)$ and $I$ is a radical ideal, $y \in I$.

Suppose now that $y \in I$. Then, every prime ideal containing $I$ explodes in $D\left[y^{-1}\right]$, and thus $I D\left[y^{-1}\right]=D\left[y^{-1}\right]$. Therefore, the same happens to $\left(D:_{D} x\right)$, and so $x \in D\left[y^{-1}\right]$ (with the same calculation of (1), just backwards).

Let now $\mathcal{U}:=\left\{\mathcal{B}\left(z^{-1}\right) \mid z \in I\right\}$.

Claim 2: $\mathcal{U}$ is an open cover of $\mathcal{B}(x) \cap \mathrm{Over}_{\mathrm{qr}}(D)$.

Let $T \in \mathcal{B}(x) \cap \operatorname{Over}_{\mathrm{qr}}(D)$ : then, $1 \in\left(T:_{T} x\right)=\left(D:_{D} x\right) T$, and thus there are $d_{1}, \ldots, d_{n} \in\left(D:_{D} x\right), t_{1}, \ldots, t_{n} \in T$ such that $1=$ $d_{1} t_{1}+\cdots+d_{n} t_{n}$. For every $i$, there is a $w_{i} \in D$ such that $w_{i}^{-1} \in T$ 
and $w_{i} t_{i} \in D$; let $w:=w_{1} \cdots w_{n}$. Then, $w$ is invertible in $T$, and thus $D\left[w^{-1}\right] \subseteq T$, that is, $T \in \mathcal{B}\left(w^{-1}\right)$; moreover,

$$
w=d_{1} w t_{1}+\cdots+d_{n} w t_{n} \in d_{1} D+\cdots+d_{n} D \subseteq\left(D:_{D} x\right) \subseteq I,
$$

and so $\mathcal{B}\left(w^{-1}\right) \in \mathcal{U}$. Therefore, $\mathcal{U}$ is a cover of $\mathcal{B}(x) \cap \operatorname{Over}_{\mathrm{qr}}(D)$.

Claim 3: there are no finite subsets of $\mathcal{U}$ that cover $\mathcal{B}(x) \cap \operatorname{Over}_{\mathrm{qr}}(D)$.

Consider a finite subset $\mathcal{U}_{0}:=\left\{\mathcal{B}\left(z_{1}^{-1}\right), \ldots, \mathcal{B}\left(z_{n}^{-1}\right)\right\}$ of $\mathcal{U}$, for some $z_{1}, \ldots, z_{n} \in I$. In particular, $\operatorname{rad}\left(z_{i} D\right) \subseteq I$ for every $I$; moreover, $\operatorname{rad}\left(z_{i} D\right) \neq I$ since $I$ is not the radical of any principal ideal. It follows that for every $i$ there is a prime ideal $P_{i}$ containing $z_{i}$ but not $I$. By prime avoidance, there is an $y \in I \backslash\left(P_{1} \cup \cdots \cup P_{n}\right)$; in particular, $D\left[y^{-1}\right] \in \mathcal{B}(x) \cap \operatorname{Over}_{\mathrm{qr}}(D)$.

We claim that $D\left[y^{-1}\right] \notin \mathcal{B}\left(z_{i}^{-1}\right)$ for every $i$ : indeed, $z_{i} \in P_{i}$, and $P_{i} D\left[y^{-1}\right] \neq D\left[y^{-1}\right]$. Therefore, $z_{i}$ is not invertible in $D\left[y^{-1}\right]$, and $z_{i}^{-1} \notin$ $D\left[y^{-1}\right]$. Hence, $D\left[y^{-1}\right]$ is an element of $\mathcal{B}(x) \cap \operatorname{Over}_{\mathrm{qr}}(D)$ not contained in any element of $\mathcal{U}_{0}$, which thus is not a cover.

Therefore, $\mathcal{B}(x) \cap \operatorname{Over}_{\mathrm{qr}}(D)$ is not compact; it follows that $\operatorname{Over}_{\mathrm{qr}}(D)$ is not proconstructible, as claimed.

We remark that the first implication of the previous theorem follows also from [24, Theorem 2.5] and the following Theorem 5.5.

Corollary 4.5. Let $D$ be a Noetherian domain, and let $X^{1}(D)$ be the set of height-1 prime ideals of $D$. The following are equivalent:

(i) $\operatorname{Over}_{\mathrm{qr}}(D)$ is proconstructible in $\operatorname{Over}(D)$;

(ii) $D=\bigcap\left\{D_{P} \mid P \in X^{1}(D)\right\}$ and every $P \in X^{1}(D)$ is the radical of a principal ideal.

Proof. (i $\Longrightarrow$ ii) Suppose that $\operatorname{Over}_{\mathrm{qr}}(D)$ is proconstructible.

Let $Q$ be a prime $t$-ideal, and consider $A:=\bigcap\left\{D_{P} \mid P \in \mathcal{D}(Q)\right\}$. We claim that $A \neq D$ : indeed, if $A=D$, then the map $\star: I \mapsto \bigcap\left\{I D_{P} \mid\right.$ $P \in \mathcal{D}(Q)\}$ would be a star operation of finite type (since $\mathcal{D}(Q)$ is compact) such that $Q^{\star}=D \nsubseteq Q=Q^{t}$, i.e., it would not be smaller than the $t$-operation, an absurdity. Hence, there is an $x \in A \backslash D$, and $\operatorname{rad}\left(\left(D:_{D} x\right)\right)=Q$. By Theorem $4.4, Q=\operatorname{rad}(y D)$ for some $y \in D$.

If $Q$ has not height 1, then this contradicts the Principal Ideal Theorem; thus, QSpec ${ }^{t}(D)=X^{1}(D)$, and $D=\bigcap\left\{D_{P} \mid P \in X^{1}(D)\right\}$.

(ii $\Longrightarrow$ i) Conversely, suppose that the two conditions hold; the first one implies that QSpec ${ }^{t}(D)=X^{1}(D)$ (since $X^{1}(D)$ is a compact subspace of $\operatorname{Spec}(D))$. For every $x \in K \backslash D,\left(D:_{D} x\right)$ is a proper $t$-ideal, and thus its minimal primes are $t$-ideals, i.e., have height 1 . However, $\left(D:_{D} x\right)$ has only finitely many minimal primes, say $P_{1}, \ldots, P_{n}$, and by hypothesis $P_{i}=\operatorname{rad}\left(y_{i} D\right)$ for some $y_{i} \in D$; hence, $\operatorname{rad}\left(\left(D:_{D} x\right)\right)$ is the radical of the principal ideal $y_{1} \cdots y_{n} D$. By Theorem 4.4, $\operatorname{Over}_{\mathrm{qr}}(D)$ is proconstructible. 
Corollary 4.6. Let $D$ be a Krull domain, and let $X^{1}(D)$ be the set of height-1 prime ideals of $D$. Then, the following are equivalent:

(i) $\operatorname{Over}_{\mathrm{qr}}(D)$ is proconstructible in $\operatorname{Over}(D)$;

(ii) each $P \in X^{1}(D)$ is the radical of a principal ideal;

(iii) the class group of $D$ is a torsion group.

Proof. The equivalence between (i) and (ii) follows as in the previous corollary, noting that $D=\bigcap\left\{D_{P} \mid P \in X^{1}(D)\right\}$ holds for every Krull domain; the equivalence of (ii) and (iii) follows from the proof of Theorem 1 of 32 .

\section{Sublocalizations}

Our first result about $\operatorname{Over}_{\text {sloc }}(D)$ shows a striking difference between the space of sublocalizations and the spaces we considered in the previous sections.

Proposition 5.1. Let $D$ be an integral domain. Then, $\operatorname{Over}_{\text {sloc }}(D)$ is a spectral space if and only if it is proconstructible in $\operatorname{Over}(D)$.

Proof. If $\operatorname{Over}_{\text {sloc }}(D)$ is proconstructible, then it is spectral. On the other hand, for every $x_{1}, \ldots, x_{n} \in K$, the intersection $\mathcal{B}\left(x_{1}, \ldots, x_{n}\right) \cap$ $\operatorname{Over}_{\text {sloc }}(D)$ is compact, since it has a minimum, namely the intersection of the localizations of $D$ that contain $x_{1}, \ldots, x_{n}$. Since $\left\{\mathcal{B}\left(x_{1}, \ldots, x_{n}\right) \cap\right.$ $\left.\operatorname{Over}_{\text {sloc }}(D) \mid x_{1}, \ldots, x_{n} \in K\right\}$ is a subbasis of $\operatorname{Over}_{\text {sloc }}(D)$, by Lemma 2.1 if $\operatorname{Over}_{\text {sloc }}(D)$ is spectral then it is also proconstructible in $\operatorname{Over}(D)$.

We are now tasked to study the spectrality of $\operatorname{Over}_{\text {sloc }}(D)$. To this end, we use spectral semistar operations; more precisely, we use the fact that there is a map

$$
\begin{aligned}
\pi: \operatorname{SStar}_{s p}(D) & \longrightarrow \operatorname{Over}_{\text {sloc }}(D) \\
\star & \longmapsto D^{\star}
\end{aligned}
$$

that is continuous [12, Proposition 3.2(2)] and surjective (by definition of $\left.\operatorname{Over}_{\text {sloc }}(D)\right)$. We shall use the following topological lemma.

Lemma 5.2. Let $\phi: X \longrightarrow Y$ be a continuous surjective map between two topological spaces. Suppose that:

(a) $X$ is spectral;

(b) $Y$ is $T_{0}$;

(c) there is a subbasis $\mathcal{C}$ of $Y$ such that, for every $C \in \mathcal{C}, \phi^{-1}(C)$ is compact.

Then, $Y$ is a spectral space and $\phi$ is a spectral map.

Proof. Let $\Omega:=O_{1} \cap \ldots \cap O_{m}$ be a finite intersection of elements of $\mathcal{C}$. Then, $\phi^{-1}(\Omega)=\bigcap_{i} \phi^{-1}\left(O_{i}\right)$ is compact, since $X$ is spectral and each $\phi^{-1}\left(O_{i}\right)$ is compact by hypothesis; moreover, since $\phi$ is surjective, also $\Omega_{i}=\phi\left(\phi^{-1}(\Omega)\right)$ is compact. Therefore, the set $\mathcal{C}_{0}$ of finite intersections 
of elements of $\mathcal{C}$ is a basis of compact subsets. If now $\Omega^{\prime}$ is any open and compact subset of $Y$, then $\Omega$ is a finite union of elements of $\mathcal{C}_{0}$, and thus $\phi^{-1}\left(\Omega^{\prime}\right)$ is also compact.

The claim now follows from [8, Proposition 9].

Proposition 5.3. Let $D$ be an integral domain. If $\operatorname{SStar}_{s p}(D)$ is a spectral space, then so is $\operatorname{Over}_{\text {sloc }}(D)$.

Proof. Let $\mathscr{B}:=\left\{\mathcal{B}(x) \cap \mathrm{Over}_{\text {sloc }}(D) \mid x \in K\right\}$ be the canonical subbasis of $\operatorname{Over}_{\text {sloc }}(D)$. Then,

$$
\begin{aligned}
\pi^{-1}\left(\mathcal{B}(x) \cap \operatorname{Over}_{\text {sloc }}(D)\right) & =\left\{\star \in \operatorname{SStar}_{s p}(D) \mid x \in D^{\star}\right\}= \\
& =\left\{\star \in \operatorname{SStar}_{s p}(D) \mid 1 \in x^{-1} D^{\star}\right\}= \\
& =\left\{\star \in \operatorname{SStar}_{s p}(D) \mid 1 \in\left(x^{-1} D\right)^{\star}\right\}= \\
& =\left\{\star \in \operatorname{SStar}_{s p}(D) \mid 1 \in\left(x^{-1} D \cap D\right)^{\star}\right\}= \\
& =V_{x^{-1} D \cap D} \cap \operatorname{SStar}_{s p}(D)=V_{\left(D:_{D} x\right)} \cap \operatorname{SStar}_{s p}(D)
\end{aligned}
$$

However, $V_{\left(D:_{D} x\right)} \cap \operatorname{SStar}_{s p}(D)$ is compact since it has a minimum (explicitly, $\left.s_{\mathcal{D}\left(\left(D:_{D} x\right)\right)}\right)$. Hence, the map $\pi: \operatorname{SStar}_{s p}(D) \longrightarrow \operatorname{Over}_{\text {sloc }}(D)$ satisfies the hypothesis of Lemma 5.2, and thus $\operatorname{Over}_{\text {sloc }}(D)$ is a spectral space.

However, $\operatorname{SStar}_{s p}(D)$ is not, in general, a spectral space. To avoid this problem, we restrict $\pi$ to the space $\operatorname{SStar}_{f, s p}(D)$ (which is always spectral [13, Theorem 4.6]), obtaining the map $\pi_{s}: \operatorname{SStar}_{f, s p}(D) \longrightarrow$ $\operatorname{Over}_{\text {sloc }}(D)$; analogously to the previous proof, we need to show that $\pi_{s}$ is surjective and that $\pi_{s}^{-1}\left(\mathcal{B}(x) \cap \operatorname{Over}_{\text {sloc }}(D)\right)$ is compact. We claim that $D$ being rad-colon coherent is a sufficient condition for this to happen; we need a lemma.

Lemma 5.4. Let $D$ be an integral domain, and let $\star$ be a spectral semistar operation on $D$.

(a) If $\mathcal{D}(F \cap D)$ is a compact subset of $\operatorname{Spec}(D)$ for every finitely generated fractional ideal $F$ of $D$, then $\star_{f}=\widetilde{\star}$.

(b) If $D$ is rad-colon coherent, then $D^{\star f}=D^{\widetilde{\star}}$.

Note that the equality $\star_{f}=\widetilde{\star}$ may actually fail; see [2, p.2466].

Proof. (a) Since $\star_{f}$ and $\widetilde{\star}$ are of finite type, it is enough to show that $F^{\star_{f}}=\bar{F}^{\star}$ if $F$ is finitely generated. The containment $F^{\widetilde{\star}} \subseteq F^{\star_{f}}$ always holds; suppose $x \in F^{\star_{f}}$. Then, since $F^{\star_{f}} \subseteq F^{\star}$, we have $x \in F^{\star}$. Consider $I:=x^{-1} F \cap D$. Then, $x I=F \cap x D \subseteq F$. Moreover,

$$
I^{\star}=\left(x^{-1} F \cap D\right)^{\star}=x^{-1} F^{\star} \cap D^{\star}
$$

since $\star$ is spectral, and thus $1 \in I^{\star}$. Since $x^{-1} F$ is finitely generated, by hypothesis $\mathcal{D}(I)$ is compact, and thus there is a finitely generated ideal $J$ of $D$ such that $\operatorname{rad}(I)=\operatorname{rad}(J)$; passing, if needed, to a power of $J$, we can suppose $J \subseteq I$, so that $x J \subseteq x I \subseteq F$. For any spectral operation $\sharp, \operatorname{rad}(A)=\operatorname{rad}(B)$ implies that $1 \in A^{\sharp}$ if and only if $1 \in B^{\sharp}$; 
therefore, $1 \in J^{\star}$, and thus $x \in(F: J) \subseteq F^{\widetilde{\star}}$, and $x \in F^{\widetilde{\star}}$. Hence, $\star_{f}=\widetilde{\star}$, as requested.

(b) It is enough to repeat the proof of the previous point by using $F=D$, and noting that $\mathcal{D}\left(x^{-1} D \cap D\right)$ is compact since $D$ is rad-colon coherent.

Theorem 5.5. Let $D$ be an integral domain. If $D$ is rad-colon coherent, then $\operatorname{Over}_{\text {sloc }}(D)$ is a spectral space.

Proof. Suppose $D$ is rad-colon coherent. If $T \in \operatorname{Over}_{\text {sloc }}(D)$, then there is a $\sharp \in \operatorname{SStar}_{s p}(D)$ such that $T=D^{\sharp}$; since $D$ is $D$-finitely generated, moreover, we have $D^{\sharp}=D^{\sharp f}$. By Lemma [5.4](b), $D^{\sharp f}=D^{\widetilde{\sharp}}$; but $\widetilde{\sharp} \in$ $\operatorname{SStar}_{f, s p}(D)$, and thus $\pi_{s}$ is surjective.

As in the proof of Proposition 5.3,

$$
\pi_{s}^{-1}\left(\mathcal{B}(x) \cap \operatorname{Over}_{\text {sloc }}(D)\right)=V_{\left(D:_{D} x\right)} \cap \operatorname{SStar}_{f, s p}(D),
$$

which is compact since it has a minimum $\left(s_{\mathcal{D}\left(\left(D:_{D} x\right)\right)}\right)$. Since $\operatorname{SStar}_{f, s p}(D)$ is a spectral space [13, Theorem 4.6], by Lemma $5.2 \operatorname{Over}_{\text {sloc }}(D)$ is spectral.

Corollary 5.6. If $D$ is a domain with Noetherian spectrum (in particular, if $D$ is Noetherian) then $\operatorname{Over}_{\text {sloc }}(D)$ is a spectral space.

Note that it is not hard to see that, if $\mathcal{D}(J)$ is not compact in $\operatorname{Spec}(D)$, then $V_{J} \cap \operatorname{SStar}_{f, s p}(D)$ is actually not compact; therefore, the proof of Theorem 5.5 cannot easily be further generalized.

Another natural question is whether $\pi_{s}$ is injective; however, this is usually false. For example, if $\Delta$ is any subset of $\operatorname{Spec}(D)$ containing the $t$-spectrum, then $\pi_{s}\left(s_{\Delta}\right)=D$. Thus, $\pi_{s}$ does not give a way to "represent" $\operatorname{Over}_{\text {sloc }}(D)$ like $\operatorname{Spec}(D)$ does for $\operatorname{Loc}(D)$ and $\mathcal{S}(D)$ for $\operatorname{Over}_{\mathrm{qr}}(D)$. To circumvent this problem, we shall use, instead of the whole spectrum, the $t$-spectrum; note that $\operatorname{QSpec}^{t}(D)$ is a proconstructible subspace of $\operatorname{Spec}(D)$ [5, Proposition 2.5], so a spectral space, and thus the space $\mathcal{X}\left(\operatorname{QSpec}^{t}(D)\right)$ is defined and spectral.

Consider the map

$$
\begin{aligned}
\pi_{t}: \mathcal{X}\left(\operatorname{QSpec}^{t}(D)\right) & \longrightarrow \operatorname{Over}_{\text {sloc }}(D) \\
\Delta & \longmapsto D^{s_{\Delta}} .
\end{aligned}
$$

Note that, if $D$ is rad-colon coherent, $\pi_{t}$ is continuous and spectral, since it is the composition of the spectral inclusion $\mathcal{X}\left(\mathrm{QSpec}^{t}(D)\right) \hookrightarrow$ $\mathcal{X}(D)$ ([15, Proposition 4.1], noting the inclusion $\operatorname{QSpec}^{t}(S) \hookrightarrow \operatorname{Spec}(D)$ is spectral since QSpec ${ }^{t}(D)$ is proconstructible), the homeomorphism $\mathcal{X}(D) \longrightarrow \operatorname{SStar}_{f, s p}(D)$ and the map $\pi_{s}: \operatorname{SStar}_{f, s p}(D) \longrightarrow \operatorname{Over}(D)$ (which is spectral by Lemma 5.2 and the proof of Theorem 5.5).

We first show that, using $\pi_{t}$, we do not lose anything.

Proposition 5.7. Let $D$ be an integral domain. Then: 
(a) for any $\Delta, \Lambda \in \mathcal{X}(D)$, if $\Delta \cap \operatorname{QSpec}^{t}(D)=\Lambda \cap \operatorname{QSpec}^{t}(D)$ then $\pi_{s}\left(s_{\Delta}\right)=\pi_{s}\left(s_{\Lambda}\right)$

(b) $\pi_{s}\left(\operatorname{SStar}_{f, s p}(D)\right)=\pi_{t}\left(\boldsymbol{\mathcal { X }}\left(\operatorname{QSpec}^{t}(D)\right)\right)$.

Proof. It is enough to show that, for every $\Delta \in \mathcal{X}(D), \pi_{s}(\Delta)=\pi_{s}\left(\Delta_{0}\right)$, where $\Delta_{0}:=\Delta \cap \operatorname{QSpec}^{t}(D)$. Let $T:=\pi_{s}\left(s_{\Delta}\right)$; then, since $\Delta$ is a proconstructible subset of $\operatorname{Spec}(D)$, also $\Delta_{0}$ is proconstructible. In particular, $\Delta_{0}$ is compact and closed by generizations relative to $\operatorname{QSpec}^{t}(D)$, and so it belongs to $\mathcal{X}\left(\mathrm{QSpec}^{t}(D)\right)$. We claim that $T=\pi_{t}\left(\Delta_{0}\right)$.

Indeed, let $P \in \Delta$. Then, $t_{P}: I D_{P} \mapsto I^{t} D_{P}$ is a star operation of finite type on $D_{P}$ (see [26]), and $Q D_{P}$ is a maximal $t_{P}$-ideal if and only if $Q$ is maximal among the $t$-prime ideals contained in $P$. Hence, $D_{P}=\bigcap\left\{D_{Q} \mid Q \subseteq P, Q=Q^{t}\right\}$, and

$$
T=\bigcap\left\{D_{Q} \mid Q=Q^{t}, Q \subseteq P \text { for some } P \in \Delta\right\} .
$$

The set of primes on the right hand side is exactly $\Delta_{0}$. Therefore, $T=\pi_{t}\left(\Delta_{0}\right) \in \pi_{t}\left(\mathcal{X}\left(\operatorname{QSpec}^{t}(D)\right)\right)$, and (a) is proved.

Moreover, this also shows that $\pi_{s}\left(\operatorname{SStar}_{f, s p}(D)\right) \subseteq \pi_{t}\left(\mathcal{X}\left(\operatorname{QSpec}^{t}(D)\right)\right)$; since the other inclusion is obvious, (b) holds.

The $t$-spectrum is much less redundant than $\operatorname{Spec}(D)$ : indeed, if $D=$ $\bigcap\left\{D_{P} \mid P \in \Delta\right\}$ for some compact $\Delta \subseteq \mathrm{QSpec}^{t}(D)$, then $\Delta$ must contain the $t$-maximal ideals, since $t$ is the biggest star operation of finite type. In general, $\pi_{t}$ is not always injective; however, when this happens then $\pi_{t}$ is also a homeomorphism, as the next proposition shows.

Proposition 5.8. Let $D$ be a rad-colon coherent domain. Then, the following are equivalent:

(i) $\pi_{t}$ is a homeomorphism;

(ii) $\pi_{t}$ is injective;

(iii) if $\Delta, \Lambda \in \mathcal{X}(D)$ are such that $\pi_{s}\left(s_{\Delta}\right)=\pi_{s}\left(s_{\Lambda}\right)$, then $\Delta \cap$ $\operatorname{QSpec}^{t}(D)=\Lambda \cap \operatorname{QSpec}^{t}(D)$.

Proof. The implication ( $\mathrm{i} \Longrightarrow$ ii) is obvious; the equivalence between (ii) and (iii) follows from Proposition 5.7.

Suppose now that $\pi_{t}$ is injective; then, $\pi_{t}$ is bijective (since it is also surjective by Theorem 5.5 , being $D$ rad-colon coherent), continuous and spectral. Clearly, if $\Delta \supseteq \Lambda$ then $\pi_{t}(\Delta) \subseteq \pi_{t}(\Lambda)$. Conversely, suppose $\pi_{t}(\Delta) \subseteq \pi_{t}(\Lambda)$ : then, $T:=\bigcap\left\{D_{P} \mid P \in \Delta\right\} \subseteq \bigcap\left\{D_{Q} \mid Q \in \Lambda\right\}$, and thus $T \subseteq D_{Q}$ for every $Q \in \Lambda$. Hence, $\pi_{t}(\Delta)=\pi_{t}(\Delta \cup \Lambda)$, and by the injectivity of $\pi_{t}$ is must be $\Delta=\Delta \cup \Lambda$, i.e., $\Lambda \subseteq \Delta$. Therefore, $\pi_{t}$ is also an order isomorphism (in the order induced by the respective topologies of $\mathcal{X}\left(\operatorname{QSpec}^{t}(D)\right)$ and $\left.\mathrm{Over}_{\text {sloc }}(D)\right)$; by [25, Proposition 15], $\pi_{t}$ is a homeomorphism.

A prime ideal $P$ of $D$ is well-behaved if $P D_{P}$ is $t$-closed in $D_{P}$ [34]; this is equivalent to $D_{P}$ being a $\mathrm{DW}$-domain, i.e., to the fact that, 
on $D_{P}$, the $w$-operation coincides with the identity (this follows from [29, Proposition 2.2]). A domain is called well-behaved if every $t$-prime ideal is well-behaved; examples of well-behaved domains are Noetherian domains, Krull domains and domains where every $t$-prime ideal has height 1 .

Proposition 5.9. Let $D$ be an integral domain. Then, $D$ is well-behaved if and only if the map $\pi_{t}: \mathcal{X}\left(\operatorname{QSpec}^{t}(D)\right) \longrightarrow \mathrm{Over}_{\text {sloc }}(D)$ is injective.

Proof. Suppose $\pi_{t}$ is injective, and let $P \in \operatorname{QSpec}^{t}(D)$ and $\Delta:=$ $\operatorname{QSpec}^{t}\left(D_{P}\right)$. Then, $\Delta$ is compact (being proconstructible in $\operatorname{Spec}\left(D_{P}\right)$ ), and thus $\Delta \cap D:=\{Q \cap D \mid P \in \Delta\}$ is a compact subspace of QSpec $^{t}(D)$, since it is the continuous image of $\Delta$ under the canonical map $\operatorname{Spec}\left(D_{P}\right) \longrightarrow \operatorname{Spec}(D)$. If $P D_{P} \notin \Delta$, then $P \notin \Delta \cap D$; however,

$$
\pi_{t}(\Delta \cap D)=\bigcap\left\{D_{Q \cap D} \mid Q \in \Delta\right\}=\bigcap\left\{\left(D_{P}\right)_{Q} \mid Q \in \Delta\right\}=D_{P},
$$

with the last equality coming from the properties of the $t$-spectrum. If we denote by $\Lambda_{1}$ the closure in the inverse topology of $\operatorname{QSpec}^{t}(D)$ of $\Delta \cap D$, and by $\Lambda_{2}$ the closure of $(\Delta \cap D) \cup\{P\}$, we have thus $\pi_{t}\left(\Lambda_{1}\right)=\pi_{t}\left(\Lambda_{2}\right)$ while $\Lambda_{1} \neq \Lambda_{2}$, against the injectivity of $\pi_{t}$.

On the other hand, suppose $D$ is well-behaved. Suppose $\pi_{t}(\Delta)=$ $\pi_{t}(\Lambda)=: T$ for some $\Delta, \Lambda \in \mathcal{X}\left(\operatorname{QSpec}^{t}(D)\right), \Delta \neq \Lambda$, and let $P \in \Delta \backslash \Lambda$. By [7, Lemma 2.4], the subspace $\left\{D_{Q} \mid Q \in \Lambda\right\} \subseteq$ Over $(D)$ is compact; then,

$$
D_{P}=D_{P} T=D_{P} \bigcap_{Q \in \Lambda} D_{Q}=\bigcap_{Q \in \Lambda} D_{P} D_{Q}
$$

with the last equality coming from [17, Corollary 5]. The family $\left\{D_{P} D_{Q} \mid\right.$ $Q \in \Lambda\}$ is again compact [17, Lemma 4]; thus, $\star: I \mapsto \bigcap_{Q \in \Lambda} I D_{P} D_{Q}$ is a finite-type spectral semistar operation such that $D^{\star}=D_{P}$, and thus it restricts to a finite-type star operation $\star^{\prime}$ on $D_{P}$. Since $P D_{P}$ is $t$-closed, and $\star^{\prime}$ is of finite type, $\left(P D_{P}\right)^{\star^{\prime}}$ must be equal to $P D_{P}$; however,

$$
P^{\star^{\prime}}=P^{\star}=\bigcap_{Q \in \Lambda} P D_{Q} D_{P}=\bigcap_{Q \in \Lambda} D_{Q} D_{P}=D_{P},
$$

since $P \nsubseteq Q$ for every $Q \in \Lambda$. This is a contradiction, and $\pi_{t}$ is injective.

\section{Remark 5.10.}

(1) There are examples of integral domains that are not well-behaved (see [34, Section 2] or [1, Example 1.4]), and thus $\pi_{t}$ is not always injective.

(2) It would be tempting to substitute the space $\mathcal{X}\left(\operatorname{QSpec}^{t}(D)\right)$ with $\mathcal{X}(\Delta)$, where $\Delta$ is the set of well-behaved $t$-prime ideals of $D$. However, $\Delta$ may not be compact and thus, a fortiori, may not be a spectral space. For example, consider a domain $D$ and 
a prime ideal $Q$ that is a maximal $t$-ideal (that is, $P$ is maximal among the ideals $I$ such that $I=I^{t}$ ) but not well-behaved. (An explicit example is $E+X E_{S}[X]$, where $E$ is the ring of entire functions, $X$ is an indeterminate and $S$ is the set of finite products of elements of the form $Z-\alpha$, as $\alpha$ ranges in $\mathbb{C}$; see [33, Example 2.6, Section 4.1 and Proposition 4.3].) Let $\Lambda$ be the set of prime ideals that are associated to some principal ideal; then, $P \in \Lambda$ if and only if $P$ is minimal over the ideal $\left(b D:_{D} a D\right)$, for some $a, b \in D$.

Since a principal ideal is t-closed, so is $\left(b D:_{D} a D\right)=\frac{b}{a} D \cap D$; moreover, a minimal prime over a $t$-ideal is again a $t$-ideal, and thus $\Lambda \subseteq \operatorname{QSpec}^{t}(D)$. Moreover, if $P \in \Lambda$ then $P D_{P}$ will be associated to a principal ideal of $D_{P}$ (if $P$ is minimal over $\left(b D:_{D}\right.$ $a D)$, then $P D_{P}$ is minimal over $\left(b D:_{D} a D\right) D_{P}=\left(b D_{P}:_{D_{P}}\right.$ $\left.\left.a D_{P}\right)\right)$. Hence, each prime of $\Lambda$ is well-behaved, and $\Lambda \subseteq \Delta$.

By [4], we have $D=\bigcap\left\{D_{P} \mid P \in \Lambda\right\}$, and thus also $D=$ $\bigcap\left\{D_{P} \mid P \in \Delta\right\}$. If $\Delta$ were compact, it would define a finitetype star operation $\star: I \mapsto \bigcap\left\{I D_{P} \mid P \in \Delta\right\}$ such that $Q^{\star}=$ $D$. On the other hand, we should have $\star \leq t$ and thus $Q^{\star} \subseteq$ $Q^{t}=Q$, a contradiction. Hence, $\Delta$ is not compact.

Recall that a domain is $v$-coherent if, for any ideal $I,(D: I)=(D$ : $J$ ) for some finitely generated ideal $J$.

Corollary 5.11. Let $D$ be a v-coherent domain. Then, $\pi_{t}$ is injective.

Proof. Since $D$ is $v$-coherent, $\left(I D_{Q}\right)^{t}=I^{t} D_{Q}$ for every ideal $I$ of $D$ [26, proof of Proposition 4.6] and every $Q \in \operatorname{Spec}(D)$; thus, if $P \in$ $\operatorname{QSpec}^{t}(D)$ then $\left(P D_{P}\right)^{t}=P^{t} D_{P}=P D_{P}$. By Proposition [5.9, $\pi_{t}$ is injective.

\section{Flat overrings}

The space $\operatorname{Over}_{\text {flat }}(D)$ of flat overrings of $D$ is much more mysterious than $\operatorname{Over}_{\mathrm{qr}}(D)$ and $\mathrm{Over}_{\text {sloc }}(D)$, and we are not able to characterize when it is spectral or proconstructible. The main theorem of this section is the following partial result.

Proposition 6.1. Let $D$ be an integral domain. Then, $\operatorname{Over}_{\text {flat }}(D)$ is a proconstructible subspace of $\operatorname{Over}(D)$ if and only if $\operatorname{Over}_{\text {flat }}(D) \cap$ $\mathcal{B}\left(x_{1}, \ldots, x_{n}\right)$ is compact for every $x_{1}, \ldots, x_{n} \in K$.

Proof. If $\operatorname{Over}_{\text {flat }}(D)$ is proconstructible, the compactness of $\operatorname{Over}_{\text {flat }}(D) \cap$ $\mathcal{B}\left(x_{1}, \ldots, x_{n}\right)$ follows like in the proof of Proposition 5.1.

Suppose that the compactness property holds, and let $x_{1}, \ldots, x_{n} \in$ $K$. Consider the canonical subbasis $\mathcal{S}:=\{\mathcal{B}(x) \cap X \mid x \in K\}$ of $X:=\operatorname{Over}_{\text {flat }}(D)$. By [10, Proposition 3.3] and [19, Theorem 8] (or [10, Corollary 2.17]), we need to show that, for every ultrafilter $\mathscr{U}$ on $X$, the ring $A_{\mathscr{U}}:=\{x \in K \mid \mathcal{B}(x) \cap X \in \mathscr{U}\}$ is flat. 
Take $a_{1}, \ldots, a_{n} \in D, x_{1}, \ldots, x_{n} \in A_{\mathscr{U}}$ such that $a_{1} x_{1}+\cdots+a_{n} x_{n}=0$. For all $C \in \operatorname{Over}_{\text {flat }}(D) \cap \mathcal{B}\left(x_{1}, \ldots, x_{n}\right)$, by the equational characterization of flatness (see e.g. [28, Theorem 7.6] or [9, Corollary 6.5]) there are $b_{j k}^{(C)} \in D, y_{k}^{(C)} \in C$ such that

$$
\begin{cases}0=a_{1} b_{1 k}^{(C)}+\cdots+a_{n} b_{n k}^{(C)} & \text { for all } k \\ x_{i}=b_{i 1}^{(C)} y_{1}^{(C)}+\cdots+b_{i N}^{(C)} y_{N}^{(C)} & \text { for all } i\end{cases}
$$

Let $\Omega(C):=\mathcal{B}\left(y_{1}^{(C)}, \ldots, y_{n_{C}}^{(C)}\right)$. Then, the family of the $\Omega(C)$ is an open cover of $\operatorname{Over}_{\text {flat }}(D) \cap \mathcal{B}\left(x_{1}, \ldots, x_{n}\right)$. Hence, there is a finite subcover $\left\{\Omega\left(C_{1}\right), \ldots, \Omega\left(C_{n}\right)\right\}$; by the properties of ultrafilters, it follows that $\Omega\left(C_{j}\right) \in \mathscr{U}$ for some $j$. Thus, $y_{i}^{\left(C_{j}\right)} \in A_{\mathscr{U}}$ for all $i$; then, (2) holds in $A_{\mathscr{U}}$. Hence, applying again the equational criterion, $A_{\mathscr{U}}$ is flat.

Corollary 6.2. Let $D$ be an integral domain such that $\operatorname{Over}_{\text {flat }}(D)=$ $\operatorname{Over}_{\text {sloc }}(D)$. Then, $\mathrm{Over}_{\text {flat }}(D)$ is a proconstructible subset of $\mathrm{Over}(D)$. In particular, $D$ is rad-colon coherent.

Proof. It is enough to note that $\operatorname{Over}_{\text {sloc }}(D) \cap \mathcal{B}\left(x_{1}, \ldots, x_{n}\right)$ has always a minimum, and apply Proposition 6.1.

Example 6.3. The space of flat overrings can be spectral even if it is not proconstructible.

Let $K$ be a field, and let $D:=K\left[\left[X^{2}, X^{3}, X Y, Y\right]\right]$; that is, $D$ is the set of the power series in two variables over $K$ without the monomial corresponding to $X$. Then, $D$ is a two-dimensional local Noetherian domain; its integral closure is $A:=K[[X, Y]]=D[X]$, which is also equal to the intersection of the localizations at the height-1 primes of $D$. (In particular, $A$ is a local sublocalization of $D$ that is not a localization.) By Corollary [5.11, it is easy to see that the sublocalizations of $D$ are $D$ itself and the intersections $T(\Delta):=\bigcap\left\{D_{P} \mid P \in \Delta\right\}$, as $\Delta$ ranges among the subsets of $X^{1}(D):=\{P \in \operatorname{Spec}(D) \mid P$ has height 1$\}$.

A power series $\phi:=\sum_{i, j \geq 0} a_{i j} X^{i} Y^{j}$ is invertible in $A$ if and only if $a_{00} \neq 0$; hence, if $\phi \in A$ is not invertible then $\phi^{2} \in D$. Since every height-1 prime ideal of $A$ is principal (being $A$ a unique factorization domain) and the canonical map $\operatorname{Spec}(A) \longrightarrow \operatorname{Spec}(D)$ is surjective, every height-1 prime ideal of $D$ is the radical of a principal ideal (if $P=Q \cap D$, for $Q \in \operatorname{Spec}(A), Q=\phi A$, then $P$ is the radical of $\left.\phi^{2} D\right)$. Hence, $T(\Delta)$ is a quotient ring of $D$ for every $\Delta \subsetneq X^{1}(D)$; in particular, they are all flat. Hence, $\operatorname{Over}_{\mathrm{qr}}(D)=\operatorname{Over}_{\text {flat }}(D)$ is spectral; however, $\left(D:_{D} X\right)$ is equal to the maximal ideal of $D$, which cannot be the radical of a principal ideal since it is of height 2. By Theorem 4.4, $\operatorname{Over}_{\mathrm{qr}}(D)$ (and so $\operatorname{Over}_{\text {flat }}(D)$ ) is not proconstructible.

The space $\operatorname{Over}_{\text {flat }}(D)$ is, however, amenable to generalizations. Indeed, if $R$ is a ring and $M$ is an $R$-module, then the set $\operatorname{SMod}_{R}(M)$ of $R$-submodules of $M$ can be endowed with a topology (called the Zariski 
topology) whose basic open sets are of the form

$$
\mathcal{D}\left(x_{1}, \ldots, x_{n}\right):=\left\{N \in \operatorname{SMod}_{R}(M) \mid x_{1}, \ldots, x_{n} \in N\right\},
$$

as $x_{1}, \ldots, x_{n}$ vary in $M$. Under this topology, $\operatorname{SMod}_{R}(M)$ is a spectral space [31, Example 2.2(2)]; moreover, if $D$ is an integral domain with quotient field $K$, then the Zariski topology on $\operatorname{Over}(D)$ is exactly the restriction of the Zariski topology on $\operatorname{SMod}_{D}(K)=\mathbf{F}(D)$, and $\operatorname{Over}(D)$ is proconstructible in $\mathbf{F}(D)$.

We can consider on $\operatorname{SMod}_{R}(M)$ the subspace $\operatorname{SModFlat}_{R}(M)$ consisting of all flat $R$-submodules of $M$. Surprisingly, in many cases spectrality and proconstructibility of $\operatorname{SModFlat}_{R}(M)$ are equivalent.

Proposition 6.4. Let $R$ be a ring and $M$ be an $R$-module; suppose that $R$ is an integral domain or that $M$ is torsion-free. Then, $\operatorname{SModFlat}_{R}(M)$ is a spectral space if and only if it is proconstructible in $\operatorname{SMod}_{R}(M)$.

Proof. Clearly if $\operatorname{SModFlat}_{R}(M)$ is proconstructible in $\operatorname{SMod}_{R}(M)$ then it is spectral.

Conversely, suppose that $Y:=\operatorname{SModFlat}_{R}(M)$ is spectral. By Lemma 2.1, $Y$ is proconstructible if and only if $\Omega \cap Y$ is compact for every $\Omega$ in some subbasis of $\operatorname{SMod}_{R}(M)$; since $\mathcal{D}\left(x_{1}, \ldots, x_{n}\right)=\mathcal{D}\left(x_{1}\right) \cap \cdots \cap \mathcal{D}\left(x_{n}\right)$ for every $x_{1}, \ldots, x_{n} \in M$, we can consider the subbasis $\{\mathcal{D}(x) \cap Y \mid x \in$ $M\}$. By definition, $\mathcal{D}(x) \cap Y:=\{N \in Y \mid x \in Y\}$.

Let $x \in M$. If $x$ has no torsion (so, in particular, if $M$ is torsion-free), then the principal submodule $\langle x\rangle$ is isomorphic to $R$, which is flat; thus, $\mathcal{D}(x) \cap Y$ has a minimum, namely $\langle x\rangle$, and $\mathcal{D}(x) \cap Y$ is compact. On the other hand, if $R$ is an integral domain, then every flat $R$-module is torsion-free [3, I.2, Proposition 3]; thus, if $x$ has torsion then no module containing $x$ can be flat, and so $\mathcal{D}(x) \cap Y$ must be empty (and in particular compact).

In all the cases considered, it follows that $\operatorname{SModFlat}_{R}(M)$ is proconstructible in $\operatorname{SMod}_{R}(M)$.

Corollary 6.5. Let $D$ be an integral domain with quotient field $K$, and suppose that $D$ is not rad-colon coherent. Then, $\operatorname{SModFlat}_{D}(K)$ is not a spectral space.

Proof. The space Over $(D)$ is proconstructible in $\operatorname{SMod}_{D}(K)$ [31, Example 2.2(5)], and thus $\operatorname{Over}_{\text {flat }}(D)$ is proconstructible in $\operatorname{Over}(D)$ if and only if it is proconstructible in $\operatorname{SMod}_{D}(K)$. If $\operatorname{SModFlat}_{D}(K)$ were spectral, by Proposition 6.4, it would follow that it is proconstructible in $\operatorname{SMod}_{D}(K)$; thus, also the intersection $\operatorname{Over}(D) \cap \operatorname{SMod}_{D}(K)=$ $\operatorname{Over}_{\text {flat }}(D)$ would be proconstructible in $\operatorname{SMod}_{D}(K)$.

However, if $D$ is not rad-colon coherent then $\operatorname{Over}_{\text {flat }}(D)$ is not proconstructible in Over $(D)$ (Proposition 3.4); hence, SModFlat $_{D}(K)$ cannot be spectral. 


\section{DARIO SPIRITO}

\section{REFERENCES}

[1] D. D. Anderson, Gyu Whan Chang, and Muhammad Zafrullah. Integral domains of finite $t$-character. J. Algebra, 396:169-183, 2013.

[2] D. D. Anderson and Sylvia J. Cook. Two star-operations and their induced lattices. Comm. Algebra, 28(5):2461-2475, 2000.

[3] Nicolas Bourbaki. Commutative Algebra. Chapters 1-7. Elements of Mathematics (Berlin). Springer-Verlag, Berlin, 1989. Translated from the French, Reprint of the 1972 edition.

[4] J. W. Brewer and W. J. Heinzer. Associated primes of principal ideals. Duke Math. J., 41:1-7, 1974.

[5] Paul-Jean Cahen, Alan Loper, and Francesca Tartarone. Integer-valued polynomials and Prüfer v-multiplication domains. J. Algebra, 226(2):765-787, 2000.

[6] Jean Dieudonné and Alexander Grothendieck. Éléments de géométrie algébrique. IV. Étude locale des schémas et des morphismes de schémas. I. Inst. Hautes Études Sci. Publ. Math., (20):259, 1964.

[7] David E. Dobbs, Richard Fedder, and Marco Fontana. Abstract Riemann surfaces of integral domains and spectral spaces. Ann. Mat. Pura Appl. (4), 148:101-115, 1987.

[8] David E. Dobbs and Marco Fontana. Kronecker function rings and abstract Riemann surfaces. J. Algebra, 99(1):263-274, 1986.

[9] David Eisenbud. Commutative Algebra, volume 150 of Graduate Texts in Mathematics. Springer-Verlag, New York, 1995. With a view toward algebraic geometry.

[10] Carmelo A. Finocchiaro. Spectral spaces and ultrafilters. Comm. Algebra, 42(4):1496-1508, 2014.

[11] Carmelo A. Finocchiaro, Marco Fontana, and K. Alan Loper. The constructible topology on spaces of valuation domains. Trans. Amer. Math. Soc., 365(12):6199-6216, 2013.

[12] Carmelo A. Finocchiaro, Marco Fontana, and Dario Spirito. New distinguished classes of spectral spaces: a survey. In Multiplicative Ideal Theory and Factorization Theory: Commutative and Non-Commutative Perspectives. Springer Verlag, 2016.

[13] Carmelo A. Finocchiaro, Marco Fontana, and Dario Spirito. Spectral spaces of semistar operations. J. Pure Appl. Algebra, 220(8):2897-2913, 2016.

[14] Carmelo A. Finocchiaro, Marco Fontana, and Dario Spirito. Topological properties of semigroup primes of a commutative ring. Beitr. Algebra Geom., 58(3):453-476, 2017.

[15] Carmelo A. Finocchiaro, Marco Fontana, and Dario Spirito. The upper Vietoris topology on the space of inverse-closed subsets of a spectral space and applications. Rocky Mountain J. Math., to appear.

[16] Carmelo A. Finocchiaro and Dario Spirito. Some topological considerations on semistar operations. J. Algebra, 409:199-218, 2014.

[17] Carmelo A. Finocchiaro and Dario Spirito. Topology, intersections and flat modules. Proc. Amer. Math. Soc., 144(10):4125-4133, 2016.

[18] Marco Fontana, James A. Huckaba, and Ira J. Papick. Prüfer Domains, volume 203 of Monographs and Textbooks in Pure and Applied Mathematics. Marcel Dekker Inc., New York, 1997.

[19] Marco Fontana and K. Alan Loper. The patch topology and the ultrafilter topology on the prime spectrum of a commutative ring. Comm. Algebra, 36(8):2917-2922, 2008.

[20] Robert Gilmer. Multiplicative Ideal Theory. Marcel Dekker Inc., New York, 1972. Pure and Applied Mathematics, No. 12. 
[21] Robert Gilmer and Jack Ohm. Integral domains with quotient overrings. Math. Ann., 153:97-103, 1964.

[22] Sarah Glaz. Commutative Coherent Rings, volume 1371 of Lecture Notes in Mathematics. Springer-Verlag, Berlin, 1989.

[23] William Heinzer and Jack Ohm. An essential ring which is not a $v$ multiplication ring. Canad. J. Math., 25:856-861, 1973.

[24] William Heinzer and Moshe Roitman. Well-centered overrings of an integral domain. J. Algebra, 272(2):435-455, 2004.

[25] Melvin Hochster. Prime ideal structure in commutative rings. Trans. Amer. Math. Soc., 142:43-60, 1969.

[26] Evan G. Houston, Abdeslam Mimouni, and Mi Hee Park. Integral domains which admit at most two star operations. Comm. Algebra, 39(5):1907-1921, 2011.

[27] B. G. Kang. Prüfer $v$-multiplication domains and the ring $R[X]_{N_{v}}$. J. Algebra, 123(1):151-170, 1989.

[28] Hideyuki Matsumura. Commutative Ring Theory, volume 8 of Cambridge Studies in Advanced Mathematics. Cambridge University Press, Cambridge, 1986. Translated from the Japanese by M. Reid.

[29] Abdeslam Mimouni. Integral domains in which each ideal is a $W$-ideal. Comm. Algebra, 33(5):1345-1355, 2005.

[30] Bruce Olberding. Noetherian spaces of integrally closed rings with an application to intersections of valuation rings. Comm. Algebra, 38(9):3318-3332, 2010.

[31] Bruce Olberding. Topological aspects of irredundant intersections of ideals and valuation rings. In Multiplicative Ideal Theory and Factorization Theory: Commutative and Non-Commutative Perspectives. Springer Verlag, 2016.

[32] Bronislaw Wajnryb and Abraham Zaks. On the flat overrings of an integral domain. Glasgow Math. J., 12:162-165, 1971.

[33] Muhammad Zafrullah. The $D+X D_{S}[X]$ construction from GCD-domains. $J$. Pure Appl. Algebra, 50(1):93-107, 1988.

[34] Muhammad Zafrullah. Well behaved prime t-ideals. J. Pure Appl. Algebra, 65(2):199-207, 1990.

[35] Oscar Zariski. The reduction of the singularities of an algebraic surface. Ann. of Math. (2), 40:639-689, 1939.

[36] Oscar Zariski. The compactness of the Riemann manifold of an abstract field of algebraic functions. Bull. Amer. Math. Soc., 50:683-691, 1944.

Dipartimento di Matematica e Fisica, Università degli Studi "Roma TRE", Roma, ItALY

E-mail address: spirito@mat.uniroma3.it 